\title{
THE ILL-POSED HELE-SHAW MODEL AND THE STEFAN PROBLEM FOR SUPERCOOLED WATER ${ }^{1}$
}

BY

\author{
EMMANUELE DI BENEDETTO AND AVNER FRIEDMAN
}

\begin{abstract}
The Hele-Shaw flow of a slow viscous fluid between slightly separated plates is analyzed in the ill-posed case when the fluid recedes due to absorption through a core $G$. Necessary and sufficient conditions are given on the initial domain occupied by the fluid to ensure the existence of a solution. Regularity of the free boundary is established in certain rather general cases.

Similar results are obtained for the analogous parabolic version, which models the one-phase Stefan problem for supercooled water.
\end{abstract}

Introduction. The Hele-Shaw flow arises when a slow incompressible viscous fluid is moving between slightly separated plates. After certain approximations in the Navier-Stokes equations, the pressure $p=p(x, y)$ satisfies

$$
\Delta p=0 \text { in the fluid. }
$$

If we denote the projection of the moving boundary of the fluid on the plane by $f(x, y, t)=0$, then

$$
p=0, \quad \frac{\partial f}{\partial t}=\nabla p \cdot \nabla f \quad \text { on } f=0
$$

A domain $G$ (the "core") surrounded by the fluid is given so that the pressure can be increased by injection of further fluid or decreased by suction of the fluid along $\partial G$, causing the fluid blob to advance or recede:

$$
\begin{array}{ll}
\frac{\partial p}{\partial \nu}=-Q & \text { along } \partial G(\text { pressure is increased }) \\
\frac{\partial p}{\partial \nu}=Q & \text { along } \partial G(\text { pressure is decreased })
\end{array}
$$

where $\nu$ is the outward normal to $\partial G$ and $Q>0$.

The model $(0.1),(0.2)$ has been proposed by Saffman-Taylor [24] and McLeanSaffman [20], who studied the displacement of one viscous fluid by another in an infinite strip. Richardson [22, 23] studied the problem $(0.1)-(0.3)$ with injection from a finite number of sources.

Received by the editors January 10, 1983.

1980 Mathematics Subject Classification. Primary 35K65, 35R35, 76D99.

${ }^{\prime}$ This work is partially supported by National Science Foundation Grants NSF 82-02100, 48-296-80 and MCS-8300293. 
One of the interests in the Hele-Shaw model is its mathematical analogy to the flow of viscous fluid in porous medium. It also appears in the theory of electrochemical machining (McGeough-Rasmussen [19]) after suitable scaling; it was studied by variational inequalities methods by Elliott [8].

Elliott and Janovsky [9] and Čižek and Janovsky [6] reduced the problem (0.1)-(0.3) to a variational inequality with a parameter $t$, and established existence and uniqueness. Numerical work was carried out by Crowley [7].

However, in the case of the boundary condition (0.4) (instead of (0.3)) the character of the problem is entirely different. As pointed by Elliott and Ockendon $[10$, p. 162] numerical work indicates that the problem is ill-posed and experimental evidence shows that initial small protuberances of the fluid's boundary grow into lengthening fingers. (For the boundary condition $(0.3)$ there is the reverse phenomenon, namely, initial protuberances of the free boundary decay as time increases.) Furthermore, finite time "blow up" occurs for suction problems (Richardson [22]).

The one-phase Stefan problem for supercooled water consists of finding a temperature $\theta(x, t)$ and a free boundary $\Phi(x, t)=0\left(x=\left(x_{1}, x_{2}, x_{3}\right)\right)$ such that

$$
\begin{gathered}
c \theta_{t}-\Delta \theta=0 \quad \text { in the water }(c>0) \\
\theta=0, \quad \partial \Phi / \partial t=\nabla \theta \cdot \nabla \Phi \quad \text { on } \Phi=0 ;
\end{gathered}
$$

further, if the water surrounds a core $G$, then

$$
\partial \theta / \partial \nu=Q \text { on } \partial G, Q \geqslant 0
$$

finally $\theta$ satisfies an initial condition

$$
\theta(x, 0)=\theta_{0}(x) \leqslant 0 .
$$

Notice that the conditions $(0.6),(0.7)$ are the same as $(0.2),(0.4)$. In the standard one-phase Stefan problem one prescribes $\theta_{0} \geqslant 0, Q \leqslant 0$ and, consequently (by the maximum principle) $\theta>0$ and the free boundary is advancing in time. In the supercooled problem, $Q \geqslant 0$ and then $\theta<0$ and the free boundary is receding.

The problem $(0.5)-(0.8)$ was studied in the literature only in the case $n=1$ and, even then, global existence (in time) for classical solutions was established only under some restrictive assumptions on $\theta_{0}$; see van Moerbecke [21], Friedman [13], Jensen [18] (some properties of the free boundary were derived by Friedman-Jensen [16] and Fasano-Primicerio [11]). The case $n=1$ is discussed in Remark 7.4 in more detail.

Notice that $(0.5)-(0.7)$ reduce to $(0.1),(0.2),(0.4)$ if $c=0$. Thus the Hele-Shaw model (with (0.4)) may be considered as the one-phase Stefan problem for supercooled water with zero specific heat.

In this paper we study both the ill-posed Hele-Shaw problem $(0.1),(0.2),(0.4)$ and the Stefan problem for supercooled water $(0.5)-(0.8)$. The methods we use for the two problems are interrelated.

For the Hele-Shaw problem we establish the existence of a weak solution (roughly) if and only if the initial domain $\Omega$ belongs to a certain class $(\mathcal{H})$ of domains; uniqueness does not hold in general. We further show that any fingering configuration can arise from a suitable initial domain $\Omega$ whose boundary is smooth 
and nearly spherical; this explains then the fingering phenomenon mentioned above. We also derive regularity results for the weak solution.

For the Stefan problem (0.5)-(0.8) we show that a weak solution exists (for all $t>0$ ) if and only if $\Omega$ belongs to some class which depends on $\theta_{0}$; here again the solution is generally not unique. This result is established under the assumption that $Q>0, \int^{\infty} Q d t<\infty$, and the solution tends to zero as $t \rightarrow \infty$.

For the ill-posed Hele-Shaw model there is a "terminal phase" $\xi$ of the solution as it disappears in finite time $T$; the same is true for the supercooled problem (however the terminal phase might occur at a finite or infinite time). The function $\xi$ represents a limiting "mushy" region for the latter problem. In case $Q=0$ and $\xi=0$ we show that the supercooled water has a finite extinction time, for which we give estimates from above and below.

We finally note that all our main results are established in $R^{n}$ with any $n \geqslant 1$.

\section{Chapter 1. The Hele-Shaw Model}

1. Classical formulation. Let $D$ and $G$ be two open sets in $R^{n}$ with $C^{2+\alpha}$ boundaries $\partial D$ and $\partial G$ (for some $0<\alpha<1$ ). We assume that $\bar{G} \subset D$ and set $\Omega=D \backslash \bar{G}$ and $\Omega_{T}=\Omega \times(0, T]$ for any $0<T<\infty$.

We wish to find $T \in(0, \infty), u: \bar{\Omega}_{T} \rightarrow \mathbf{R}$ and $\Phi: \bar{\Omega}_{T} \rightarrow \mathbf{R}$ such that, setting

$$
\Omega(t)=\{x \in \Omega ; \Phi(x, t)<0\}, \quad \partial_{0} \Omega(t)=\partial \Omega(t) \backslash \partial G,
$$

the following conditions are satisfied:

$$
\begin{gathered}
-\Delta u=0 \quad \text { in } \Omega(t), 0<t \leqslant T, \\
u=0 \quad \text { on } \partial_{0} \Omega(t), 0<t \leqslant T, \\
\partial u / \partial \nu=Q \quad \text { on } \partial G \times\{0<t \leqslant T\}, \\
\nabla_{x} u \cdot N_{x}=N_{t} \quad \text { on } \partial_{0} \Omega(t), 0<t \leqslant T, \\
u \leqslant 0 \quad \text { in } \Omega_{T}, \quad u(x, 0)<0 \quad \text { in } \Omega, \\
u(x, T) \equiv 0,
\end{gathered}
$$

where $\nu$ is the outward normal to $\partial G$ and $Q$ is a given positive number, and

$$
N_{x}=\frac{\nabla_{x} \Phi}{|\nabla \Phi|}, \quad N_{t}=\frac{\Phi_{t}}{|\nabla \Phi|} .
$$

If $u$ is a classical solution of (1.1)-(1.6) then, by the maximum principle, $\nabla_{x} u \neq 0$ on $\partial_{0} \Omega(t)$, and we can take $\Phi=u$ in the above. Then (1.4) becomes

$$
\left|\nabla_{x} u\right|^{2}=u_{t}
$$

By the maximum principle it then follows that $u_{t} \geqslant 0$; hence the free boundary is decreasing in the sense that

$$
\Omega\left(t_{1}\right) \subset \Omega\left(t_{2}\right) \text { if } t_{1}>t_{2}
$$

Notice finally that (1.5) is actually a consequence of (1.1)-(1.3) and the maximum principle. 
2. Weak formulation. Assume that $u \in C^{2,1}\left(\Omega_{T}\right)$ is a classical solution of (1.1)-(1.6) with free boundary $\Phi(x, t)=0$ of class $C^{2,1}$.

Let $\phi \in C_{0}^{\infty}\left(\Omega_{T}\right)$ be such that supp $\phi \cap(\bar{\Omega} \times\{T\})$ is empty, and denote by $\langle\cdot, \cdot\rangle$ the distribution pairing in $\Omega_{T}$. Then

$$
\begin{aligned}
\langle-\Delta u, \phi\rangle & =-\iint_{\Omega_{T}} u \Delta \phi=\iint_{[u<0]} \nabla_{x} u \cdot \nabla_{x} \phi d x d t \\
& =\int_{0}^{T} \int_{\partial_{0} \Omega(t)} \nabla_{x} u \cdot N_{x} \phi d \sigma-\iint_{[u<0]} \Delta u \cdot \phi d x d t .
\end{aligned}
$$

Using (1.1) and (1.4) we get

$$
\langle-\Delta u, \phi\rangle=\int_{\Gamma} N_{t} \phi d S
$$

where

$$
\Gamma=\bigcup_{0<t \leqslant T}\left((x, t) ; x \in \partial_{0} \Omega(t)\right)
$$

is the free boundary.

We introduce the graph

$$
H(s)= \begin{cases}1 & \text { if } s<0 \\ {[0,1]} & \text { if } s=0 \\ 0 & \text { if } s>0\end{cases}
$$

and compute

$$
\left\langle\frac{\partial}{\partial t} H(u), \phi\right\rangle=\left\langle H(u),-\phi_{t}\right\rangle=-\iint_{[u<0]} \phi_{t} d x d t=-\int_{\Gamma} N_{t} \phi d S .
$$

Comparing with (2.1) we conclude that

$$
\frac{\partial}{\partial t} H(u)-\Delta u \ni 0 \text { in } \mathscr{D}^{\prime}\left(\Omega_{T}\right) .
$$

Introduce a new unknown function

$$
v(x, t)=\int_{t}^{T} u(x, \tau) d \tau
$$

and observe that if $u$ is a classical solution then, by virtue of $(1.8), H(u(x, t))=$ $H(v(x, t))$. Integrating (2.2) from $t$ to $T$ we then obtain

$$
-\Delta v \in H(v)-\xi(x) \quad \forall t \in[0, T]
$$

where $\xi(x) \in H(u(x, T))$ is to be determined.

In order to determine $T$ we integrate (2.3) over $\Omega(t)$ for $0<t<T$. This gives

$$
Q(T-t)|\partial G|-\int_{\partial_{0} \Omega(t)} \nabla_{x} v \cdot N_{x} d \sigma=\int_{\Omega}[H(v(x, t))-\xi(x)] d x
$$

where $|\partial G|$ denote the $H^{1}(\partial G)$ measure of $\partial G$ and $H(v)$ is interpreted as a selection of a function from the graph $H(v)$. 
Next we observe that

$$
\int_{\partial_{0} \Omega(t)} \nabla_{x} v \cdot N_{x} d \sigma=0
$$

Indeed, writing $\partial_{0} \Omega(t)$ as a union of two parts

$$
\Gamma_{1}(t)=\partial_{0} \Omega(t) \cap \Omega, \quad \Gamma_{2}(t)=\partial_{0} \Omega(t) \cap \partial D
$$

we have

$$
\nabla_{x} v=0 \text { on } \Gamma_{1}(t)
$$

(since $v$ takes interior maximum on $\Gamma_{1}(t)$ ); further, on $\Gamma_{2}(t)$,

$$
\left|\nabla_{x} v\right|^{2}=\left|\int_{t}^{T} \nabla_{x} u(x, \tau) d \tau\right|^{2} \leqslant(T-t) \int_{t}^{T}\left|\nabla_{x} u\right|^{2} d \tau=(T-t) \int_{t}^{T} u_{t}=0
$$

by (1.7) and the fact that $u=0$ on $\Gamma_{2}(t) \subset \partial D$.

Using (2.5), we obtain from (2.4)

$$
Q|\partial G|(T-t)=\int_{\Omega(t)}(H(v)-\xi) d x \quad \forall 0<t<T,
$$

and, taking $t \rightarrow 0$,

$$
Q|\partial G| T=\int_{\Omega}(1-\xi(x)) d x
$$

The formulas derived for $v$, under the assumption that $T, u, \Phi$ represent a classical solution will now be used to define the concept of a weak solution.

We seek $T \in(0, \infty), \xi: \Omega \rightarrow[0,1]$ and $v: \bar{\Omega}_{T} \rightarrow \mathbf{R}$ such that the following conditions hold:

$$
\begin{gathered}
v \leqslant 0, \\
v \in C^{0}\left([0, T] ; H^{2}(\Omega)\right), \\
-\Delta v \in H(v)-\xi \quad \text { a.e. in } \Omega, \forall t \in[0, T], \\
v=0 \quad \text { on } \partial D \times[0, T], \\
\frac{\partial v}{\partial \nu}=Q(T-t) \quad \text { on } \partial G \times\{t\}, 0<t<T, \\
v(x, T) \equiv 0, \\
Q|\partial G| T=\int_{\Omega}(1-\xi(x)) d x, \\
v(x, 0)<0 \quad \text { in } \Omega .
\end{gathered}
$$

Equation (2.9) has to be interpreted in the sense that

$$
-\Delta v=\eta(x, t)-\xi(x) \text { a.e. in } \Omega_{T}
$$

where $\eta$ is a selection out of the graph $H(v)$. Notice that $(2.11)$ is understood here in a weak sense; however we shall prove later on that $v_{x}$ is Hölder continuous in $\bar{\Omega} \times(0, T)$, and thus (2.11) will be satisfied in the classical sense. 
Definition 2.1. A triple $(T, \xi, v)$ satisfying (2.7)-(2.14) is called a weak solution of the Hele-Shaw problem (1.1)-(1.6); $\xi$ is called the terminal phase.

Notice that (2.13) is the "balance of energy" equation, and (in view of (1.8)) (2.14) means that $u(x, 0)<0$ in $\Omega$, i.e., the fluid is initially occupying the domain $\Omega$.

If a weak solution $v$ and the free boundary $\partial\{v<0\} \cap \Omega_{T}$ are sufficiently smooth then $u=-v_{t}$ is a classical solution of (1.1)-(1.6).

Notice finally that (2.9) and (2.12) imply that

$$
\xi(x)=H(v(x, T)) \text {. }
$$

\section{Existence of solutions.}

LEMMA 3.1. If $(T, \xi, v)$ is a weak solution then

$$
\frac{\partial v}{\partial \nu}(x, 0)=0 \quad \text { on } \partial D .
$$

PRoof. Integrating (2.9), for $t=0$, over $\Omega$ and using (2.11) and (2.13), we get

$$
\int_{\partial D} \frac{\partial v}{\partial \nu}(x, 0) d \sigma=0
$$

On the other hand, since $v \leqslant 0$ in $\Omega_{T}$ and $v=0$ on $\partial D$, we have $\partial v / \partial \nu \geqslant 0$ on $\partial D$, and (3.1) follows.

From Lemma 3.1 we deduce

COROllary 3.2. If there exists a weak solution $(T, \xi, v)$ of the Hele-Shaw model then the function $V(x)=v(x, 0)$ is a solution of the variational inequality

$$
\left.\begin{array}{c}
-\Delta V \leqslant 1-\xi \\
V \leqslant 0 \\
V(-\Delta V-1+\xi)=0
\end{array}\right\} \quad \text { a.e. in } R^{n} \backslash G,
$$

and

$$
\Omega=\{x ; V(x)<0\} .
$$

Definition 3.1. Suppose $\xi=\chi_{\Sigma}$ where $\Sigma$ is a disjoint union of open sets $\Sigma_{i}$ in $R^{n} \backslash \bar{G}$ such that each $\partial \Sigma_{i}$ contains an open subset of $\partial G$; then we say that $\xi$ belongs to class $\left(S_{0}\right)$.

Definition 3.2. Suppose $\xi \in C^{\alpha}\left(R^{n} \backslash G\right)$ (for some $0<\alpha<1$ ), $\xi$ has compact support, $0 \leqslant \xi(x)<1$; then we say that $\xi$ belongs to class $\left(S_{1}\right)$.

Definition 3.3. Suppose $\xi=\xi_{0}+\xi_{1}$ where $\xi_{0} \in\left(S_{0}\right), \xi_{1} \in\left(S_{1}\right)$ and $0 \leqslant \xi(x) \leqslant$ 1; then we say that $\xi$ belongs to class $(S)$.

Notice that $\xi \equiv 0$ belongs to $(S)$.

Let $\xi \in(S)$ and consider the variational inequality (3.2), (3.3). It is well known that this problem has a unique solution $V$, and $V$ has compact support $[15$, p. 9]; further,

$$
V \in W^{2, p}\left(R^{n} \backslash G\right) \quad \forall 1 \leqslant p<\infty, \quad V \in W^{2, \infty}(K),
$$

for any open set $K$ with $\bar{K} \subset R^{n} \backslash(G \cup \partial \Sigma)$, where $\xi=\xi_{0}+\xi_{1}, \xi_{i} \in\left(S_{i}\right), \xi_{0}=\chi_{\Sigma}$. 
Definition 3.4. For any $\xi \in(S)$ and $\lambda>0$, denote by $\Omega(\xi, \lambda)$ the open set $\{V<0\}$ where $V$ is the solution of the variational inequality (3.2), (3.3) with $Q T=\lambda$. The class of all $\Omega(\xi, \lambda)$ will be denoted by $(\mathcal{H})$.

In view of Corollary 3.2, if there exists a weak solution of the Hele-Shaw model for an initial domain $\Omega$, then $\Omega$ must belong to $(\mathcal{H})$.

We shall now prove the converse.

THEOREM 3.3. If $\xi \in(S)$ and $\Omega=\Omega(\xi, \lambda) \in(\mathcal{H})$ then for any $0<T<\infty$ there exists a unique weak solution $(T, \xi, v)$ of the Hele-Shaw model with $Q=\lambda / T$, and $v$ satisfies the following properties:

(i) $0 \leqslant v_{t} \leqslant C(C$ constant $)$;

(ii) $|\Delta v| \leqslant 2$;

(iii) $\nabla_{x} v$ is Hölder continuous in $x$, with exponent $\alpha$, uniformly in $\Omega_{T}, \forall \alpha \in(0,1)$;

(iv) $\nabla_{x} v$ is Hölder continuous in $t$, with exponent $\beta$, uniformly in $\Omega_{T}, \forall \beta \in\left(0, \frac{1}{2}\right)$;

(v) if $\xi=\xi_{0}+\xi_{1}$ with $\xi_{i} \in\left(S_{i}\right), \xi_{0}=\chi_{\Sigma}$ then, if $\Sigma \neq \varnothing$,

$$
v(x, t)<0 \text { if } x \in \Sigma, 0 \leqslant t<T
$$

if, further, $\partial \Sigma \in C^{2}$ then

$$
v(x, t)<0 \text { if } x \in \bar{\Sigma}, 0 \leqslant t<T .
$$

Proof. The proof of uniqueness is standard since $H(v)$ is monotone decreasing. To prove existence we introduce $C^{\infty}$ functions $\beta_{\varepsilon}(s)(\varepsilon>0)$ satisfying

$$
\begin{gathered}
\beta_{\varepsilon}^{\prime}(s) \leqslant 0, \quad \beta_{\varepsilon}^{\prime \prime}(s) \leqslant 0 \quad \forall s \in \mathbf{R}, \\
\beta_{\varepsilon}(s)=1 \quad \text { if } s<-\varepsilon, \quad \beta_{\varepsilon}(0)=-1, \\
\beta_{\varepsilon}(s) \rightarrow-\infty \quad \text { if } s>0, \varepsilon \rightarrow 0,
\end{gathered}
$$

and a domain $\tilde{D}$ with $C^{2+\alpha}$ boundary such that $\tilde{D} \supset \bar{D}$. Set $\tilde{\Omega}=\tilde{D} \backslash G$ and consider, for each $t \in[0, T]$ the "penalized" problem

$$
\begin{aligned}
-\Delta v^{\varepsilon} & =\beta_{\varepsilon}\left(v^{\varepsilon}\right)-\xi^{\varepsilon}, \\
\frac{\partial}{\partial \nu} v^{\varepsilon} & =Q(T-t) \quad \text { on } \partial G, \\
v^{\varepsilon} & =0 \text { on } \partial \tilde{D}
\end{aligned}
$$

where $\xi^{\varepsilon}$ is a mollification of $\xi$. By the maximum principle we find that $v^{\varepsilon} \leqslant 0$ and, by a standard argument we can also estimate the minimum of $\beta_{\varepsilon}\left(v_{\varepsilon}\right)$, obtaining $-1 \leqslant \beta_{\varepsilon}\left(v^{\varepsilon}\right) \leqslant 1$. Hence

$$
\left|\Delta v^{\varepsilon}\right| \leqslant 2
$$

The function $z=\partial v^{\varepsilon} / \partial t$ satisfies

$$
\begin{aligned}
-\Delta z & =\beta_{\varepsilon}^{\prime}\left(v^{\varepsilon}\right) z \quad \text { in } \tilde{\Omega} \\
\partial z / \partial \nu & =-Q \quad \text { on } \partial G, \\
z & =0 \quad \text { on } \partial \tilde{D} .
\end{aligned}
$$

Hence, by the maximum principle, $z \geqslant 0$. It follows that $-\Delta z \leqslant 0$ in $\tilde{\Omega}$ and then, by comparison, $z \leqslant C$ where $C$ is independent of $\varepsilon$. Thus

$$
0 \leqslant \partial v^{\varepsilon} / \partial t \leqslant C .
$$


Taking $\varepsilon \rightarrow 0$ we obtain a solution $v$ of $(2.7)-(2.11)$ with $\Omega$ replaced by $\tilde{\Omega}$ and with $v(x, 0)<0$ in $\Omega$. Since $v_{t} \geqslant 0$ (by (3.8)), $v$ actually satisfies (2.7)-(2.11) in $\Omega$. Next, (2.13) follows by integrating $-\Delta v=1-\xi$ over $\Omega$ (for $t=0$ ) and using (2.11).

From (3.7), (3.8) and the fact that the $v^{\varepsilon}$ are bounded in $L^{2}\left(0, T ; W^{2, p}(\Omega)\right)$ $\forall p \in[1, \infty]$ we see that (i), (ii) hold and then (iii) follows by elliptic estimates. We next prove (iv).

Let $x, y$ be any two points in $\Omega$ with $\overline{x y}$ in $\Omega$ and let $l$ be the direction of $\overrightarrow{x y}$, and $0<t_{1}<t_{2}<T$. Using (iii) we have

$$
\begin{gathered}
\left|\int_{x}^{y}\left[\frac{\partial}{\partial l} v\left(z, t_{2}\right)-\frac{\partial}{\partial l} v\left(y, t_{1}\right)\right] d l\right|=|x-y|\left|\frac{\partial v}{\partial l}\left(\eta, t_{2}\right)-\frac{\partial}{\partial l} v\left(y, t_{1}\right)\right| \\
\geqslant|x-y|\left\{\left|\frac{\partial v}{\partial l}\left(y, t_{2}\right)-\frac{\partial v}{\partial l}\left(y, t_{1}\right)\right|-\gamma_{1}|x-y|^{\alpha}\right\}
\end{gathered}
$$

where $\eta$ is a point in the interval $\overline{x y}$.

On the other hand

$$
\begin{aligned}
\int_{x}^{y}\left[\frac{\partial v}{\partial l}\left(z, t_{2}\right)-\frac{\partial v}{\partial l}\left(y, t_{1}\right)\right] d z= & \int_{x}^{y}\left[\frac{\partial v}{\partial l}\left(z, t_{2}\right)-\frac{\partial v}{\partial l}\left(z, t_{1}\right)\right] d z \\
& +\int_{x}^{y}\left[\frac{\partial v}{\partial l}\left(z, t_{1}\right)-\frac{\partial v}{\partial l}\left(y, t_{1}\right)\right] d z \\
& \equiv I_{1}+I_{2} .
\end{aligned}
$$

In view of (iii) $\left|I_{2}\right| \leqslant \gamma|x-y|^{1+\alpha}$. As for the first integral we have

$$
\begin{aligned}
\left|I_{1}\right| & \leqslant\left|v\left(y, t_{2}\right)-v\left(y, t_{1}\right)\right|+\left|v\left(x, t_{2}\right)-v\left(x, t_{1}\right)\right| \\
& \leqslant 2\left(\sup _{\Omega_{T}} v_{t}\right)\left|t_{2}-t_{1}\right| .
\end{aligned}
$$

Putting these estimates in (3.9), we get

$$
|x-y|\left|\frac{\partial v}{\partial l}\left(y, t_{2}\right)-\frac{\partial v}{\partial l}\left(y, t_{1}\right)\right| \leqslant \gamma\left(|x-y|^{1+\alpha}+\left|t_{2}-t_{1}\right|\right),
$$

or

$$
\left|\frac{\partial v}{\partial l}\left(y, t_{2}\right)-\frac{\partial v}{\partial l}\left(y, t_{1}\right)\right| \leqslant \gamma\left(|x-y|^{\alpha}+\frac{\left|t_{2}-t_{1}\right|}{|x-y|}\right) .
$$

For a fixed $y$ in $\Omega$ we choose $x$ such that $\overline{x y}$ is in $\Omega$ and the right-hand side of (3.10) is minimized; this gives

$$
\left|\frac{\partial v}{\partial l}\left(y, t_{2}\right)-\frac{\partial v}{\partial l}\left(y, t_{1}\right)\right| \leqslant \gamma\left|t_{2}-t_{1}\right|^{\alpha /(1+\alpha)},
$$

which completes the proof of (iv) since $\alpha$ is an arbitrary number in $(0,1)$.

It remains to prove (2.12) and (v).

LEMMA 3.4. There holds

$$
v(x, t)<0 \quad \text { if } x \in \Sigma, 0 \leqslant t<T .
$$


Proof. Since $H(v)-\xi=H(v)-1 \leqslant 0$ if $x \in \Sigma$,

$$
-\Delta v \leqslant 0, \quad v \leqslant 0 \text { if } x \in \Sigma .
$$

Hence for each $t \in[0, T)$ either (3.11) holds or else $v \equiv 0$ in some component $\Sigma_{i}$ of $\Sigma$. Since however

$$
\frac{\partial v}{\partial \nu}=Q(T-t) \neq 0 \quad \text { on } \partial \Sigma_{i} \cap \partial G
$$

and $\partial \Sigma_{i} \cap \partial G$ is a nonempty open portion of $\partial G$, the second latter case cannot arise.

LEMMA 3.5. If $\partial \Sigma \in C^{2}$ then

$$
v(x, t)<0 \text { if } x \in \bar{\Sigma}, 0 \leqslant t<T .
$$

Indeed otherwise $v\left(x_{0}, t_{0}\right)=0$ for some $0 \leqslant t_{0}<T, x_{0} \in \partial \Sigma$. By the strong maximum principle we have that

$$
\frac{\partial}{\partial \nu} v\left(x_{0}, t_{0}\right) \neq 0
$$

which is impossible since $v\left(x, t_{0}\right)$ take an interior maximum at $x=x_{0}$.

LEMMA 3.6. There holds

$$
H(v(x, t))-\xi(x) \geqslant 0 \quad \text { if } 0 \leqslant t<T .
$$

Proof. By Lemma 3.4, (3.13) certainly holds if $x \in \Sigma$. In $\Omega \backslash \bar{\Sigma}, \xi=\xi_{1} \in\left(S_{1}\right)$ and therefore

$$
1-\xi_{1}>0 \text { on the free boundary in } \Omega \backslash \bar{\Sigma} \text {. }
$$

This nondegeneracy condition implies (see $[1,4$ or 15, p. 156]) that

$$
\operatorname{meas}\left(\partial_{0} \Omega(t) \backslash \Sigma\right)=0 .
$$

Now, on the set $\{v(t)<0\} \backslash \Sigma$ clearly $H(v(t))-\xi=1-\xi_{1} \geqslant 0$, whereas in the interior of the set $\{v(t)=0\} \backslash \Sigma$

$$
H(v(t))-\xi=-\Delta v(t)=0 .
$$

In view of (3.15) it then follows that

$$
H(v(t))-\xi \geqslant 0 \quad \text { a.e. in } \Omega .
$$

From Lemma 3.6 it follows that $v(t)$ satisfies $-\Delta v \geqslant 0$ in $\mathscr{Q}^{\prime}(\Omega)$. Since $v$ is continuous in $\bar{\Omega} \times[0, T], v(T)$ is also superharmonic. Recalling that $v \leqslant 0$ and

$$
\frac{\partial v(T)}{\partial \nu}=0 \quad \text { on } \partial G, \quad v(T)=0 \quad \text { on } \partial D
$$

we deduce that $v(T) \equiv 0$. Thus (2.12) holds. Finally (v) follows from Lemmas 3.4 and 3.5 .

REMARK 3.1. If $\xi=\xi_{0}+\xi_{1}, \xi_{i} \in\left(S_{i}\right), \xi_{0}=\chi_{\Sigma}$ then, since $-\Delta V \leqslant 0$ on $\Sigma$, the maximum principle yields (cf. the proof of Lemma 3.4) $V<0$ in $\Sigma$, i.e., $\Omega(\xi, \lambda) \supset \Sigma$. Further if $\partial \Sigma \in C^{2}$, then $V<0$ on $\bar{\Sigma}$ (cf. the proof of Lemma 3.5), so that $\Omega(\xi, \lambda) \supset \bar{\Sigma}$. 
REMARK 3.2. A domain $\Omega$ in ( $\mathcal{H}$ ) may have the form $\Omega(\xi, \lambda)=\Omega\left(\xi^{\prime}, \lambda^{\prime}\right)$ for some $\xi \neq \xi^{\prime}$, which means that two distinct weak solutions may develop from the same initial domain $\Omega$, having the same $Q$. We give such an example in case $n=2$,

$$
D=\left\{0 \leqslant r<R_{0}\right\}, \quad G=\left\{0 \leqslant r<\rho_{0}\right\} \quad \text { with } \rho_{0}<R_{0},
$$

taking $\xi=\chi_{\Sigma}, \Sigma=\{0 \leqslant r<\rho\}$ with any $\rho_{0}<\rho<R_{0}$, and

$$
T=\frac{1}{2 \rho_{0} Q}\left(R_{0}^{2}-\rho^{2}\right) \quad(\text { in accordance with }(2.13)) .
$$

We get a 1-parameter family of solutions (with parameter $\rho$ )

$$
\begin{gathered}
V(r, t)=\rho_{0} Q(T-t) \log \frac{r}{\phi(t)}-\frac{1}{4}\left[\phi^{2}(t)-\max \left(r^{2}, \rho^{2}\right)\right]+\frac{1}{2} \rho^{2} \log \frac{\phi(t)}{\max (r, \rho)}, \\
\phi^{2}(t)=\rho^{2}+2 \rho_{0} Q(T-t)
\end{gathered}
$$

with free boundary $r=\phi(t)$.

4. Regularity of the free boundary; the fingering phenomenon. We shall establish regularity of the free boundary under some conditions on $G$ and $\xi$. For simplicity consider first the case where $\xi=\chi_{\Sigma}$ and

$$
G \text { is a ball } B_{\rho_{0}}=\left\{x ;|x|<\rho_{0}\right\},
$$

$\partial \Sigma$ is of class $C^{2}$ and it is star-shaped

with respect to any point $x_{0}$ in $B_{\varepsilon}$,

for some $\varepsilon \in\left(0, \rho_{0}\right)$.

THEOREM 4.1. If (4.1), (4.2) hold and $\xi=\chi_{\Sigma}$, then, for the solution constructed in Theorem 3.3, the free boundary $\partial_{0} \Omega(t)$ is star-shaped with respect to any point $x_{0}$ in some ball $B_{\delta}$ with $0<\delta<\varepsilon$; further, $\Gamma \equiv \cup_{0 \leqslant t \leqslant T}\left\{(x, t) ; x \in \partial_{0} \Omega(t)\right\}$ can locally be represented in the form

$$
x_{i}=f\left(x_{1}, \ldots, x_{i-1}, x_{i+1}, \ldots, x_{n}, t\right)
$$

where $f$ is analytic in the $x_{j}$ and uniformly Hölder continuous in $t$, with any exponent $\beta \in\left(0, \frac{1}{2}\right)$.

PROOF. For simplicity let us prove the star-shaped property for $n=2$ (the proof for general $n$ is similar). In polar coordinates

$$
\frac{1}{r}\left(r v_{r}\right)_{r}+\frac{1}{r^{2}} v_{\theta \theta}=\xi-1 \quad \text { in }\{v(t)<0\} .
$$

Differentiating in $r$ we get

$$
\left(r v_{r}\right)_{r r}+\frac{1}{r}\left(r v_{r}\right)_{r}+\frac{1}{r^{2}}\left(r v_{r}\right)_{\theta \theta}=r \frac{\partial \xi}{\partial r}+2(\xi-1) .
$$

Since $\Sigma$ is star-shaped, $\partial \xi / \partial r \leqslant 0$ in $\mathscr{Q}^{\prime}[v(t)<0]$; since $\Sigma \subset\{v(t)<0\}$, also $\xi-1 \leqslant 0$. Consequently,

$$
\left(r v_{r}\right)_{r r}+\frac{1}{r}\left(r v_{r}\right)_{r}+\frac{1}{r^{2}}\left(r v_{r}\right)_{\theta \theta} \leqslant 0 \quad \text { in } \mathscr{D}^{\prime}[v(t)<0]
$$


On the free boundary we have $v_{r}=0$; further,

$$
v_{r}=Q>0 \text { on } \partial G \text {. }
$$

Applying the maximum principle to $r v_{r}$ we find that $v_{r}>0$ in $[v(t)<0]$. This establishes the star-shaped property with respect to the origin.

Similarly we can establish the star-shaped property with respect to any point $x_{0}$ in a ball $B_{\delta}$ with $\delta$ small enough so that $\delta<\varepsilon$ and

$$
\frac{\partial}{\partial \rho} v>0 \text { on } \partial G
$$

where $\rho$ is the radial direction with $x_{0}$ as center.

The star-shaped property with respect to any point $x_{0}$ in $B_{\delta}$ implies Lipschitz continuity of $\partial_{0} \Omega(t)$ (for each $t$ ). By Lemma 3.5 we also have

$$
-\Delta v=1 \text { in } \Omega(t) \text {-neighborhood of } \partial_{0} \Omega(t) .
$$

We can therefore apply a general theorem of Caffarelli $[1,2]$ to deduce that $\partial_{0} \Omega(t)$ is locally analytic, for each $t$.

Since $v_{t} \in L^{\infty}\left(\Omega_{T}\right)$,

$$
\|v(\cdot, t+h)-v(\cdot, t)\|_{L^{x}(\Omega)} \leqslant C|h| .
$$

By a general stability result of the free boundary [3] we then deduce the Hölder continuity in $t$, for any exponent $\beta \in\left(0, \frac{1}{2}\right)$.

REMARK 4.1. Theorem 4.1 can be extended to the case where $\partial G$ is not a sphere but close to a sphere in the $C^{2+\alpha}$ sense; for this will ensure that (4.5) still holds. The theorem extends also to $\xi=\chi_{\Sigma}+\xi_{1}$ where $\Sigma$ is as in (4.2) and $\partial \xi_{1} / \partial \rho \leqslant 0$ for any radial direction $\rho$ with respect to $x_{0}, x_{0} \in B_{\varepsilon}$; if $\xi_{1} \in C^{m+\alpha}$ (analytic) then $\partial_{0} \Omega(t)$ is in $C^{m+1+\alpha}$ (analytic).

REMARK 4.2. If the functions $f$ in (4.3) are in $C^{1+\beta}$ in $t$, then $u=-v_{t}$ is a classical solution of the Hele-Shaw problem.

We shall now explain the phenomenon of fingers. Let $G$ be a ball as in (4.1) and let $\lambda \equiv Q T$ be a large number. We can compare the $V$ (with a given $\xi \in(S)$ ) with radial solutions $V_{1}$ and $V_{2}$ of the variational inequalities (3.2), (3.3) corresponding to $\xi=0$ and $\xi=\chi_{B_{R} \backslash G}$, respectively, where $B_{R} \supset \operatorname{supp} \xi$. Then $V_{1}(x) \leqslant V(x) \leqslant V_{2}(x)$. We can compute the $V_{i}$ explicitly. Taking $n \geqslant 2$, we find that the free boundary $r=R_{i}$ of $V_{i}$ is given by

$$
R_{i}=c_{n} \lambda^{1 / n}(1+O(1 / \lambda))
$$

hence

$$
R_{2}-R_{1}=O\left(\lambda^{-(1-1 / n)}\right)
$$

Since the free boundary $\partial_{0} \Omega$ of $V$ lies in the shell $R_{2}<r<R_{1}$, the estimate (4.6) shows that $\partial_{0} \Omega$ satisfies a "flatness condition". Using this property in the proof of Caffarelli's theorem [2] (see also [15, §2.5]) we deduce that $\partial_{0} \Omega$ has the form

$$
r=\rho(\theta) \quad \text { with } \rho \text { analytic. }
$$


Further, since $r=\rho(\theta)$ lies in a shell of width $O\left(\lambda^{-(n-1) / n}\right)$ and since $\left|D^{2} \rho\right| \leqslant C$, using Taylor's formula we find that (cf. [15, p. 155]) $|D \rho(\theta)| \leqslant C \lambda^{-(n-1) / 2 n}$. Thus

$$
\begin{gathered}
r=\rho(\theta)=c_{n} \lambda^{1 / n}(1+g(\theta)), \quad|g(\theta)| \leqslant C \lambda^{-1}, \\
|D g(\theta)| \leqslant C \lambda^{-(n+1) / 2 n} .
\end{gathered}
$$

We summarize:

THEOREM 4.2. Given any $\xi$ in $(S)$, one can choose $Q$ and $T$ with $Q T$ sufficiently large such that there exists a weak solution of the Hele-Shaw problem with $\Sigma, Q, T$ and with $\partial_{0} \Omega$ analytic and nearly spherical in the sense of (4.7) (with $\rho(\theta)$ analytic).

REMARK 4.3. Take, in particular, $\xi=\chi_{\Sigma}, \Sigma$ in $\left(S_{0}\right)$ with any number of fingers protruding from $\partial G$. Since $\{v(t)<0\}$ is connected to $\partial G$ (by the maximum principle) and contains $\Sigma$, and since $\{v(t)<0\}$ decreases to $\Sigma$ as $t \uparrow T$, the domain $\{v(t)<0\}$ exhibits approximately the same fingers as $\Sigma$, for $t$ near $T$. Nevertheless, the initial domain is nearly spherical in the sense of (4.7).

REMARK 4.4. The methods of this chapter also yield new results in the case of Hele-Shaw with increasing pressure (or advancing fluid) considered by Elliott and Janovsky [9].

Suppose the initial domain $\Omega_{0}$ occupied by fluid has fingers. Then, after a sufficiently long time the advancing free boundary is nearly spherical; in fact, it is given by

$$
r=\rho_{t}(\theta)=C_{n} t^{1 / n}\left(1+g_{t}(\theta)\right)
$$

with

$$
\left|g_{t}(\theta)\right| \leqslant C t^{-1}, \quad\left|D_{\theta} g_{t}(\theta)\right| \leqslant C t^{-(n+1) / 2 n} .
$$

Indeed we simply compare the radial solutions and use the same analysis which led (4.7).

We finally mention that the analog of Theorem 4.1 also holds for this case; if $\partial \Omega_{0}$ is star-shaped with respect to any point in a ball $B_{\varepsilon}$, then the same is true for the free boundary $\partial_{0} \Omega(t)$ at any time $t$. The proof can be given, for instance, by first working with the one-phase Stefan problem with specific heat $c$ (for which the result is indeed valid; cf. [17]) and then taking $c \rightarrow 0$.

\section{Chapter 2. The Stefan PROBlem For SUPERCOOLED WATER}

5. Classical formulation. Let $D$ and $G$ be open sets in $\mathbf{R}^{n}$ such that $\bar{G} \subset D$, with $C^{2+\alpha}$ boundaries $\partial D$ and $\partial G$, respectively (for some $0<\alpha<1$ ). Set $\Omega=D \backslash \bar{G}$, $\Omega_{T}=\Omega \times(0, T), 0<T \leqslant \infty$. We seek $T \in(0, \infty], u: \bar{\Omega}_{T} \rightarrow \mathbf{R}$ and $\Phi: \bar{\Omega}_{T} \rightarrow \mathbf{R}$ such that if

$$
\Omega(t)=\{x \in \Omega ; \Phi(x, t)<0\}, \quad \partial_{0} \Omega(t)=\partial \Omega(t) \backslash \partial G,
$$

then the following conditions are satisfied:

$$
\begin{gathered}
u_{t}-\Delta u=0 \text { in } \underset{0<t<T}{\bigcup}(\Omega(t) \times\{t\}), \\
u=0 \text { on } \partial_{0} \Omega(t), 0<t<T,
\end{gathered}
$$




$$
\begin{gathered}
\frac{\partial u}{\partial \nu}=Q(x, t)>0 \quad \text { if } x \in \partial G, 0<t<T, \\
\nabla_{x} u \cdot N_{x}=N_{t} \quad \text { on } \partial_{0} \Omega(t), 0<t<T, \\
u(x, 0)=u_{0}(x) \quad \text { if } x \in \Omega, \quad u_{0}(x)<0 \quad \text { in } \Omega, \\
u \leqslant 0 \quad \text { in } \Omega_{T}, \\
u(x, T) \equiv 0 ;
\end{gathered}
$$

here $\nu$ is the outward normal to $\partial G, u_{0}$ and $Q$ are given functions, and

$$
N_{x}=\frac{\nabla_{x} \Phi}{|\nabla \Phi|}, \quad N_{t}=\frac{\Phi_{t}}{|\nabla \Phi|} .
$$

Notice that (5.6) is actually a consequence of the maximum principle and the other conditions; in fact $u<0$ in $[\Phi<0]$. Thus we can take $\Phi=u$ in (5.4), which reduces it to

$$
u_{t}=\left|\nabla_{x} u\right|^{2} \quad \text { on } \partial_{0} \Omega(t), 0<t<T .
$$

Consequently the domains $\Omega(t)$ are decreasing in $t$.

6. Weak formulation. Assume that $u \in C^{2,1}\left(\bar{\Omega}_{T}\right)$ is a classical solution of (5.1)-(5.7) with free boundary $\left[\Phi=0\right.$ ] of class $C^{2.1}$. For any $\phi \in C_{0}^{\infty}\left(\Omega_{T}\right)$ with supp $\phi \cap(\bar{\Omega} \times$ $\{T\})=\varnothing$ we have

$$
\begin{aligned}
\left\langle u,-\phi_{t}\right\rangle+\langle u,-\Delta \phi\rangle & =\left\langle u,-\phi_{t}\right\rangle+\iint_{[u<0]} \nabla_{x} u \cdot \nabla_{x} \phi d x d t \\
& =-\left\langle u, \phi_{t}\right\rangle+\int_{0}^{T} \int_{\partial_{0} \Omega(t)} \nabla_{x} u \cdot N_{x} \phi d \sigma-\iint_{[u<0]} \Delta u \cdot \phi d x d t \\
& =\iint_{[u<0]}\left(u_{t}-\Delta u\right) \phi d x d t+\int_{0}^{T} \int_{\partial_{0} \Omega(t)} N_{t} \phi d \sigma d t .
\end{aligned}
$$

Since

$$
\begin{aligned}
\left\langle\frac{\partial}{\partial t} H(u), \phi\right\rangle & =-\left\langle H(u), \phi_{t}\right\rangle=-\iint_{[u<0]} \phi_{t} d x d t \\
& =-\int_{0}^{T} \int_{\partial_{0} \Omega(t)} N_{t} \phi d \sigma d t \quad(H \text { as in } \S 2),
\end{aligned}
$$

we find that $\left\langle u_{t}-\Delta u, \phi\right\rangle=-\left\langle H(u)_{t}, \phi\right\rangle$, that is,

$$
\frac{\partial}{\partial t}(u+H(u))-\Delta u \ni 0 \quad \text { in } \mathscr{D}^{\prime}\left(\Omega_{T}\right) .
$$

Introduce the new unknown function

$$
v(x, t)=\int_{t}^{T} u(x, \tau) d \tau
$$

and observe that if $u$ is a classical solution and the free boundary decreases (cf. §5) then $H(u(x, t))=H(v(x, t))$. Integrating (6.1) over $(t, T)$ we then obtain

$$
u(x, T)-u(x, t)-\Delta v(x, t)=H(v(x, t))-\xi(x)
$$


where $\xi(x)$ is a selection out of the graph $H(v(x, T))$. Using (5.7) we then get

$$
v_{t}-\Delta v=H(u)-\xi(x) \text { in } \mathscr{D}^{\prime}\left(\Omega_{T}\right) .
$$

We now integrate (6.2) over $\Omega$, for any $0<t<T$, and obtain (6.3)

$$
\int_{\Omega} v_{t} d x+\int_{t}^{T} \int_{\partial G} Q(x, \tau) d \sigma d \tau-\int_{\partial_{0} \Omega(t)} \nabla_{x} v \cdot N_{x} d \sigma=\int_{\Omega}[H(v(x, t))-\xi(x)] d x .
$$

As in the Hele-Shaw case, if $u$ is a classical solution then we must have

$$
\int_{\partial_{0} \Omega(t)} \nabla_{x} v \cdot N_{x} d \sigma=0, \quad 0<t<T .
$$

Therefore, letting $t \rightarrow 0$ in (6.3) and using (5.5) we get the "balance of energy" equation

$$
\int_{0}^{T} \int_{\partial G} Q(x, \tau) d \sigma d \tau=\int_{\Omega}\left[1+u_{0}(x)-\xi(x)\right] d x,
$$

which is analogous to (2.6).

We summarize the conditions on $T, \xi$ and $v$ obtained from (5.1)-(5.7):

$$
\begin{gathered}
v \leqslant 0, \\
v \in C\left(0, \bar{T} ; L^{2}(\Omega)\right) \cap L^{2}\left(0, \bar{T} ; H^{1}(\Omega)\right) \quad \forall \bar{T} \in(0, T), \\
v_{t}-\Delta v \in H(v)-\xi \quad \text { in } \mathscr{Q}^{\prime}\left(\Omega_{T}\right), \\
v=0 \quad \text { on } \partial D \times(0, T), \\
\frac{\partial v}{\partial \nu}=\int_{t}^{T} Q(x, \tau) d \tau \quad \text { if } x \in \partial G, 0<t<T, \\
v(x, T) \equiv 0, \\
\int_{0}^{T} \int_{\partial G} Q(x, \tau) d \sigma d \tau=\int_{\Omega}\left(1+u_{0}-\xi\right) d x, \\
-\Delta v(x, 0)=1+u_{0}-\xi \quad \text { if } x \in \Omega ; v(x, 0)<0 \quad \text { in } \Omega .
\end{gathered}
$$

Notice that (6.10) is a consequence of (6.6) and the assumption that $u_{0}(x)<0$ in $\Omega$. Notice also that (6.9), (6.6) imply, if $v$ is classical solution global in time,

$$
\xi(x)=H(v(x, T)) .
$$

Definition 6.1. A triple $(T, \xi, v)$ satisfying (6.4)-(6.11) is called a weak solution; $\xi$ is called the terminal phase, and $T$ the extinction time.

7. Existence of solutions. We shall assume

$$
\begin{aligned}
& Q \in C^{2+\alpha}(\partial G) \quad \text { in } x, \text { uniformly in } t \\
& Q_{x} \in C^{\alpha}(\partial G \times[0, \infty)) \text { for some } 0<\alpha<1, \\
& 0<Q \leqslant C, \quad \int_{0}^{\infty} Q(x, t) d t \leqslant C \quad(C<\infty),
\end{aligned}
$$

and

$$
\begin{aligned}
& u_{0} \in C^{\alpha}\left(R^{n} \backslash G\right) \text { for some } 0<\alpha<1, \\
& u_{0} \text { has compact support, } \quad u_{0} \leqslant 0 .
\end{aligned}
$$


Suppose there exists a solution of (6.4)-(6.11) with $T=\infty$. If we integrate (6.11) and use (6.10), (6.8), we obtain

$$
\int_{\partial D} \frac{\partial v(x, 0)}{\partial \nu} d \sigma=0 .
$$

Since however $\partial v / \partial \nu \geqslant 0$, we conclude that $\partial v(x, 0) / \partial \nu=0$ along $\partial D$. Hence $v(x, 0)$ must coincide with the solution $V$ of the variational inequality

$$
\left.\begin{array}{c}
-\Delta V \leqslant 1+u_{0}-\xi \\
V \leqslant 0 \\
V\left(-\Delta V-1-u_{0}+\xi\right)=0
\end{array}\right\} \quad \text { a.e. in } R^{n} \backslash G,
$$

Definition 7.1. We denote by $\Omega=\Omega(\xi)$ the open set $\{V<0\}$, where $V$ is the solution of (7.3), (7.4). We denote by $\left(\mathcal{H}_{u_{0}}\right)$ the class of all $\Omega(\xi)$ when $\xi$ varies in $(S)$.

As shown above, if there is a solution of (6.4)-(6.11) with $T=\infty$ then $\Omega$ must belong to the class $\left(\mathcal{H}_{u_{0}}\right)$. We shall now prove the converse.

THEOREM 7.1. If $\xi \in(S)$ and $\Omega=\Omega(\xi) \in\left(\mathcal{H}_{u_{0}}\right)$ then there is a unique solution $(\infty, \xi, v)$ of the Stefan problem (6.4)-(6.11), and $v$ satisfies the following properties:

(i) $0 \leqslant v_{t} \leqslant C(C$ constant $)$, $v_{t}$ is subcaloric;

(ii) $|\Delta v| \leqslant C$;

(iii) $\nabla_{x} v$ is Hölder continuous in $x$, with exponent $\alpha$, uniformly in $\Omega_{\infty}, \forall \alpha \in(0,1)$;

(iv) $\nabla_{x} v$ is Hölder continuous in $t$, with exponent $\beta$, uniformly in $\Omega_{\infty}, \forall \beta \in\left(0, \frac{1}{2}\right)$;

(v) If $\xi=\xi_{0}+\xi_{1}$ with $\xi_{i} \in\left(S_{i}\right), \xi_{0}=\chi_{\Sigma}$ then, if $\Sigma \neq \varnothing$,

$$
v(x, t)<0 \text { if } x \in \Sigma, 0<t<\infty \text {; }
$$

if, further, $\partial \Sigma \in C^{2}$ then

$$
v(x, t)<0 \quad \text { if } x \in \bar{\Sigma}, 0<t<\infty ;
$$

(vi) if $\xi \equiv 0, Q \equiv 0$ and $u_{0} \in C^{2}(\bar{\Omega}), \Delta u_{0} \geqslant 0$, then $v_{t t} \leqslant 0$ in $\mathscr{D}^{\prime}\left(\Omega_{\infty}\right)$.

Proof. Let $v_{0}$ be the unique solution of the variational inequality (7.3), (7.4) (i.e., $\left.v_{0}=V\right)$. By standard results [15] there exists a unique solution $v(t)$ of the parabolic variational inequality $(6.4),(6.6)$ with boundary conditions $(6.7),(6.8)(T=\infty)$ and initial condition $v_{0}$. Since $V_{0}<0$ in $\Omega$,

$$
-\Delta v_{0}=1+u_{0}-\xi \text { in } \Omega \text {. }
$$

Integrating this equation over $\Omega$ we obtain (6.10).

To establish regularity we use the penalty method. We introduce $\beta_{\varepsilon}$ as in (3.5) and $\tilde{D}, \tilde{\Omega}=\tilde{D} \backslash \bar{G}$ where $\tilde{D} \supset \bar{D}, \partial \tilde{D} \in C^{2+\alpha}$ and consider the parabolic problem:

$$
\begin{gathered}
v_{t}^{\varepsilon}-\Delta v^{\varepsilon}=\beta_{\varepsilon}\left(v^{\varepsilon}\right)-\xi^{\varepsilon} \quad \text { in } \tilde{\Omega} \times(0, \infty), \\
\frac{\partial}{\partial \nu} v^{\varepsilon}=\int_{t}^{\infty} Q(x, \tau) d \tau \quad \text { on } \partial G \times(0, \infty), \\
v^{\varepsilon}=0 \quad \text { on } \partial \tilde{D} \times(0, \infty), \\
v^{\varepsilon}(x, 0)=v_{0}^{\varepsilon}(x) \quad \text { in } \tilde{\Omega},
\end{gathered}
$$


where $\xi^{\varepsilon}$ is a mollification of $\xi$ and $v_{0}^{\varepsilon}$ is the solution of

$$
\begin{gathered}
-\Delta v_{0}^{\varepsilon}=\beta_{\varepsilon}\left(v_{0}^{\varepsilon}\right)+u_{0}-\xi^{\varepsilon} \quad \text { in } \tilde{\Omega}, \\
\frac{\partial v_{0}^{\varepsilon}}{\partial \nu}=\int_{0}^{\infty} Q(x, \tau) d \tau \quad \text { on } \partial G, \\
v_{0}^{\varepsilon}=0 \text { on } \partial \tilde{D} .
\end{gathered}
$$

By the maximum principle we find that $v_{0}^{\varepsilon} \leqslant 0$ and, similarly, $v^{\varepsilon} \leqslant 0$. Next, considering the points where $\beta_{\varepsilon}\left(v^{\varepsilon}(x, t)\right)$ takes its minimum we find that $-1 \leqslant \beta_{\varepsilon} \leqslant 1$. Hence

$$
\left|v_{t}^{\varepsilon}-\Delta v^{\varepsilon}\right| \leqslant 2
$$

Set $z=\partial v^{\varepsilon} / \partial t$. Then

$$
z_{t}-\Delta z=\beta_{\varepsilon}^{\prime}\left(v^{\varepsilon}\right) z \text { in } \tilde{\Omega}_{\infty}
$$

and

$$
\begin{gathered}
\partial z / \partial \nu=-Q \quad \text { on } \partial G \times(0, \infty), \\
z=0 \text { on } \partial \tilde{D} \times(0, \infty), \\
z(x, 0)=-u_{0}(x) \text { on } \tilde{\Omega} .
\end{gathered}
$$

Since $\beta_{\varepsilon}^{\prime} \leqslant 0$ the maximum principle can be applied to yield

$$
\frac{\partial v^{\varepsilon}}{\partial t} \geqslant 0
$$

Consequently, from (7.6),

$$
z_{t}-\Delta z \leqslant 0 \quad \text { in } \tilde{\Omega}_{\infty}
$$

Comparing $z$ with a solution of the heat equation in $\tilde{\Omega}_{\infty}$ satisfying the same initial and boundary conditions we find that

$$
\frac{\partial v^{\varepsilon}}{\partial t} \leqslant C
$$

We now take $\varepsilon \rightarrow 0$. Since $v \equiv \lim v^{\varepsilon}$ (uniform limit) is a solution of (6.4)-(6.8) with $\Omega$ replaced by $\tilde{\Omega}$, and since $v_{t} \geqslant 0$ (by (7.7)), $v$ is actually the solution of (6.4)-(6.8) (with $\tilde{\Omega}=\Omega$ ). Further, (ii) and the first part of (i) follow from (7.5), (7.7) and (7.8). The proof of (iii), (iv) is as in $\$ 3$.

Notice that, by Minty's lemma, $\lim \beta_{\varepsilon}\left(v^{\varepsilon}\right)=H(v)$ (weak limit). Since

$$
\frac{\partial}{\partial t} \beta_{\varepsilon}\left(v^{\varepsilon}\right) \leqslant 0
$$

we then have

$$
\frac{\partial}{\partial t} H(v(x, t)) \leqslant 0 \quad \text { in } \mathscr{D}^{\prime}\left(\Omega_{\infty}\right)
$$

From this and (6.6) it follows that $v_{t}$ is subcaloric, i.e.,

$$
\left(v_{t}\right)_{t}-\Delta v_{t} \leqslant 0 \text { in } \mathscr{D}^{\prime}\left(\Omega_{\infty}\right)
$$


It remains to prove (6.9) and (v), (vi).

Since $v_{t} \geqslant 0$,

$$
-\Delta v \leqslant H(v)-\xi
$$

Hence the proofs of Lemma 3.4, 3.5 immediately extend to the present case:

$$
\begin{gathered}
v(x, t)<0 \quad \text { if } x \in \Sigma, 0<t<\infty \\
v(x, t)<0 \quad \text { if } x \in \bar{\Sigma}, 0<t<\infty \text { provided } \partial \Sigma \in C^{2} .
\end{gathered}
$$

The proof of Lemma 3.6 is based on (3.14), (3.15). The extension of (3.14) to the present case is immediate since it only requires the inequality (7.11). As for (3.15), it also extends to the present case; in fact the proof applies to any parabolic variational inequality for which the nondegeneracy condition (like (3.14) holds) (see [1 or 15, §2.9]). We conclude that

$$
H(v(x, t))-\xi(x) \geqslant 0 .
$$

Integrating (6.6) over $\Omega_{\infty}$ we find that

$$
\int_{0}^{\infty} \int_{\Omega}(H(v(x, t))-\xi(x)) d x d t<\infty .
$$

Using (7.9), (7.14) we deduce that

$$
H(v(x, t)) \downarrow \xi(x) \quad \text { as } t \uparrow \infty, \text { a.e. in } \Omega,
$$

i.e., (6.12) holds.

From (7.14) we have $v_{t}-\Delta v \geqslant 0$ in $\Omega_{\infty}$. We compare $v$ with the solution $w$ of

$$
\begin{gathered}
w_{t}-\Delta w=0 \quad \text { in } \Omega_{\infty}, \\
w=0 \quad \text { on } \partial D \times(0, \infty), \\
\frac{\partial w}{\partial \nu}=\int_{t}^{\infty} Q(x, \tau) d \tau \quad \text { on } \partial G \times(0, \infty), \\
w(x, 0)=v_{0}(x) \quad \text { if } x \in \Omega .
\end{gathered}
$$

Clearly $w \leqslant v$ in $\Omega_{\infty}$. Since however $w(x, t) \rightarrow 0$ if $x \in \Omega, t \rightarrow \infty$, the same is true for $v$; hence $\lim _{t \rightarrow \infty} v(x, t)=0$ if $x \in \Omega$, and the proof of (6.9) is complete.

The assertion (v) follows from (7.12), (7.13).

Finally, to prove (vi) we differentiate (7.6) with respect to $t$. The function $\zeta=\partial^{2} v^{\varepsilon} / \partial t^{2}$ satisfies

$$
\zeta_{t}-\Delta \zeta=\beta_{\varepsilon}^{\prime \prime}\left(v^{\varepsilon}\right) z^{2}+\beta_{\varepsilon}^{\prime}\left(v^{\varepsilon}\right) \zeta \leqslant \beta_{\varepsilon}^{\prime}\left(v^{\varepsilon}\right) \zeta
$$

and

$$
\begin{array}{cc}
\frac{\partial \zeta}{\partial \nu}=0 & \text { on } \partial G \times(0, \infty), \\
\zeta=0 & \text { on } \partial D \times(0, \infty) ;
\end{array}
$$

further,

$$
\zeta(x, 0)=\Delta z+\beta_{\varepsilon}^{\prime}\left(v^{\varepsilon}\right) z=-\Delta u_{0}-\beta_{\varepsilon}^{\prime}\left(v^{\varepsilon}\right) u_{0} \leqslant 0 \quad \text { in } \Omega
$$


since $\Delta u_{0} \geqslant 0, \beta_{\varepsilon}^{\prime} \leqslant 0, u_{0} \leqslant 0$. If $\zeta$ is bounded then the maximum principle gives $\zeta \leqslant 0$ and then, letting $\varepsilon \rightarrow 0$, (vi) follows. However, since $\zeta$ need not be bounded, we use, instead, the representation

$$
\zeta(x, t)=-\iint_{\Omega_{t}} K\left(\beta_{\varepsilon}^{\prime \prime \prime} z^{2}\right)+\int_{\Omega} K(x, t ; y, 0) \zeta(y, 0) d y
$$

where $K$ is Green's function for $\zeta_{t}-\Delta \zeta-\beta_{\varepsilon}^{\prime} \zeta$ with $K_{\nu}=0$ on $\partial G \times(0, \infty), K=0$ on $\partial D \times(0, \infty)$. To establish this representation, we first write it in $\Omega_{t} \backslash \bar{\Omega}_{\delta}, \delta>0$, and then show that, as $\delta \rightarrow 0$,

$$
\int_{\Omega} K(x, t ; y, \delta) \zeta(y, \delta) d y \rightarrow \int_{\Omega} K(x, t ; y, 0) \zeta(y, 0) d y
$$

this last step is accomplished by substituting

$$
\zeta=z_{t}=\Delta z+\beta_{\varepsilon}^{\prime} z
$$

and integrating by parts in

$$
\int_{\Omega} K(x, t ; y, \delta) \Delta z(y, \delta) d y
$$

then taking $\delta \rightarrow 0$ and integrating by parts in the reverse direction.

Remark 7.1. As in $\$ 4$ one can show that $\partial \Omega$ is star-shaped if $\xi, u_{0}$ satisfy appropriate conditions. However the proof does not carry over to $\partial_{0} \Omega(t)$, since $v_{t}$, has the wrong sign.

REMARK 7.2. It may occur that $\Omega(\xi)=\Omega\left(\xi^{\prime}\right)$ where $\xi \neq \xi^{\prime}$; this means that there will be two solutions (with different terminal phases) corresponding to the same domain $\Omega$. Consider for example the case $n=1$ and let $\xi$ be any function in $(S)$ such that

$$
\begin{gathered}
\int_{0}^{x}\left(1+u_{0}-\xi\right) d x<\lambda \quad \text { if } 0 \leqslant x<L, \lambda>0 \\
\int_{0}^{L}\left(1+u_{0}-\xi\right) d x=\lambda .
\end{gathered}
$$

Then the solution $V$ of

$$
\begin{gathered}
-V^{\prime \prime}=1+u_{0}-\xi \quad(0<x<L) \\
V^{\prime}(0)=\lambda, \quad V(L)=0
\end{gathered}
$$

satisfies $V^{\prime}(x)>0$ if $0 \leqslant x<L, V^{\prime}(L)=0$, and therefore $V(x)<0$ if $0 \leqslant x<L$. But clearly one can find, in general, many functions $\xi$ satisfying (7.15). Thus there is no uniqueness for $v$.

ReMARK 7.3. Theorem 7.1 does not extend to the case of finite time $T$. We give a counterexample with $n=1, \Omega=(0, L), Q=1$, and $\xi, u_{0}$ satisfying (7.15) with $\lambda$ small. We can evidently choose $u_{0}-\xi$ such that $V(0) \geqslant c>0$ where $c$ is a fixed positive constant independent of $\lambda$. Since for a solution $(T, \xi, v)$ we must have $T=\lambda$, whereas by continuity we have $v(0, \lambda)>c / 2>0$ if $\lambda$ is small enough, we cannot achieve the assertion $v(T) \equiv 0$. 
REMARK 7.4. If $n=1$ and

$$
\left(u_{0}-\xi\right)^{\prime} \geqslant 0
$$

then, by the maximum principle, $v_{x}(x, t) \geqslant 0$. It follows that there is a curve $x=s(t)$ such that

$$
v(x, t)<0 \text { if and only if } 0 \leqslant x<s(t) .
$$

We can now apply the method of $[15$, p. 248 , Exercise 3$]$ in order to conclude, in case $\xi \in\left(S_{0}\right)$, that $s(t)$ is Lipschitz continuous for $t>0$. It then follows by well-known arguments $[5,25]$ that $s(t)$ is $C^{\infty}$ for $t>0$; if $\xi \equiv 0$ then $s(t)$ is analytic [14].

If, in addition to (7.16), we assume that

$$
\lim _{x \rightarrow L} \frac{1+u_{0}^{\prime \prime}(x)}{u_{0}^{\prime}(x)} \text { exists, } \quad u_{0}^{\prime}(0)=0,
$$

then there is a unique classical local solution which can be obtained by the methods of nonlinear integral equations (see [12]); for this solution $s^{\prime}(t)$ is continuous up to $t=0$.

Our method provides weak solutions $v$. Their free boundaries are $C^{\infty}$ curves $x=s(t)$ for $t>0$, but we get no information on $s(t)$ for $t=0$; nor are these solutions uniquely determined (see Remark 7.2).

For the Cauchy problem existence of global classical solutions was established in $[\mathbf{2 1}, \mathbf{1 3}]$ and more generally in [18]. The results in [18], when specialized to our model, assume that $Q \equiv 0, \xi \equiv 0$ and (7.16), (7.17) hold.

8. Finite extinction time. The results of $\S 7$ extend to the case $Q \geqslant 0$ (instead $Q>0)$ provided $\xi \in\left(S_{1}\right)$. In fact, the condition $Q>0$ was needed only for establishing (7.12). Let us now consider the case

$$
Q \equiv 0, \quad \xi \equiv 0 \text {. }
$$

Then (6.10) becomes

$$
\int_{\Omega}\left(1+u_{0}\right) d x=0 .
$$

We would like to know whether $v(t)$ vanishes for $t$ sufficiently large.

THEOREM 8.1. If (8.1) holds then the solution $v$ (constructed in Theorem 7.1) has finite extinction time, i.e., $v(x, T) \equiv 0$ for some $0<T<\infty$.

Proof. Integrating (6.6) we get

so that

$$
\int_{[v(t)<0]} v_{t} d x=\operatorname{meas}[v(t)<0]
$$

$$
\left|v_{t}\right|_{L^{\infty}(\Omega)} \operatorname{meas}[v(t)<0] \geqslant \operatorname{meas}[v(t)<0] .
$$

Consequently $v(t)=0$ if

$$
\left|v_{t}\right|_{L^{\infty}(\Omega)}<1
$$


Thus it suffices to prove (8.3) for some $t<\infty$.

Differentiating (6.6) in $t$ and recalling that $(H(v))_{t} \leqslant 0$, we get, for $z=v_{t}$, the inequality

$$
z_{t}-\Delta z \leqslant 0 \text { in } \mathscr{Q}^{\prime}\left(\Omega_{\infty}\right) \text {. }
$$

Also $z=0$ on $\partial D \times(0, \infty)$ and $\partial z / \partial \nu=0$ on $\partial G \times(0, \infty)$. If $U$ satisfies the same boundary conditions and

$$
\begin{aligned}
& U_{t}-\Delta U=0 \quad \text { in } \Omega_{\infty}, \\
& U \geqslant z \quad \text { on } \Omega \times\{0\}
\end{aligned}
$$

then, on one hand,

$$
\sup _{x \in \Omega}|U(x, t)| \rightarrow 0 \quad \text { if } t \rightarrow \infty,
$$

and, on the other hand, $0 \leqslant z \leqslant U$ in $\Omega_{\infty}$, by comparison. Hence (8.3) holds for $t$ large enough.

REMARK 8.1. Theorem 8.1 clearly extends to the case where $Q(x, t)=0$ if $t \geqslant T_{0}$, for some $T_{0}<\infty$.

We shall derive estimates on the extinction time $T$. The lower bound will involve the function $\phi$ defined by

$$
\Delta \phi=0 \quad \text { in } \Omega, \quad-\partial \phi / \partial \nu=1 \quad \text { on } \partial G, \quad \phi=0 \quad \text { on } \partial D .
$$

For the upper bound we shall assume that

$$
G \text { is a ball } B_{R} \text {. }
$$

Let $\tilde{u}_{0}(r)$ be a radial function satisfying

$$
\tilde{u}_{0}(r) \leqslant u_{0}(x), \quad \tilde{u}_{0}^{\prime} \geqslant 0, \quad \Delta \tilde{u}_{0} \geqslant 0 .
$$

Denote by $\tilde{\Omega}$ the domain $\Omega(\xi)$ corresponding to $\tilde{u}_{0}$ and $\xi=0$ (then $\tilde{\Omega} \supset \Omega$ ) and by $\tilde{\phi}$ the solution of

$$
\Delta \tilde{\phi}=0 \quad \text { in } \Omega, \quad-\partial \tilde{\phi} / \partial \nu=1 \quad \text { on } \partial G, \quad \tilde{\phi}=0 \quad \text { on } \partial \tilde{D}
$$

where $\tilde{D}=\tilde{\Omega} \backslash \bar{G}$.

THEOREM 8.2. (i) There holds

$$
T \geqslant-\frac{\int_{\Omega}\left(1+u_{0}\right) \phi}{|\partial G|\left|u_{0}\right|_{\infty, \Omega}}
$$

(ii) if (8.5) holds then

$$
T \leqslant-\frac{\int_{\tilde{\Omega}}\left(1+\tilde{u}_{0}\right) \tilde{\phi}}{|\partial G|} .
$$

Proof. The function $z=v_{t}$ satisfies

$$
\begin{gathered}
z_{t}-\Delta z=\frac{\partial}{\partial t} H(v) \leqslant 0 \quad \text { in } \Omega_{\infty}, \\
\frac{\partial z}{\partial \nu}=0 \quad \text { on } \partial G \times(0, \infty), \quad z=0 \quad \text { on } \partial D \times(0, \infty), \\
z(x, 0)=-u_{0}(x) \text { in } \Omega .
\end{gathered}
$$


It follows that

$$
|z|_{L^{\infty}\left(\Omega_{\infty}\right)} \leqslant\left|u_{0}\right|_{\infty, \Omega}
$$

Multiplying (8.9) by $\phi$ and integrating over $\Omega_{T}$ we get

$$
\int_{\Omega} u_{0} \phi+\int_{0}^{T} \int_{\partial G} z d \sigma d t=-\int_{\Omega} \phi d x
$$

i.e.,

$$
\int_{0}^{T} \int_{\partial G} z d \sigma d \tau=-\int_{\Omega}\left(1+u_{0}\right) \phi d x
$$

Using (8.12) we obtain (8.7).

To prove $(8.8)$ we work with the solution $\tilde{v}(r, t)$ corresponding to $\tilde{u}_{0}(r)$. Analogously to (8.13) we have

$$
\int_{0}^{\tilde{T}} \int_{\partial G} \tilde{z} d \sigma d \tau=-\int_{\tilde{\Omega}}\left(1+\tilde{u}_{0}\right) \tilde{\phi} d x
$$

where $\tilde{T}$ is the extinction time for $\tilde{v}$.

By applying $r^{n}(\partial / \partial r)$ to

$$
\tilde{v}_{t}-\Delta \tilde{v}=1 \text { in }\{\tilde{v}<0\}
$$

and using the maximum principle we find that $\tilde{v}_{r} \geqslant 0$. Hence the free boundary of $\tilde{v}$ is given by $r=\tilde{s}(t)$ and $\tilde{v}_{r}(r, t)>0$ if $R<r<\tilde{s}(t), 0<t<\tilde{T}$. Further, $\tilde{s}(\tilde{T})=0$ for otherwise we also have $\tilde{v}_{r}(r, \tilde{T})<0$ if $R<r<\tilde{s}(\tilde{T})$, contradicting $\tilde{v}(r, \tilde{T}) \equiv 0$.

From the proof of Theorem 8.1 (see (8.3)) we have that

$$
\sup _{R \leqslant r \leqslant \tilde{s}(t)} \tilde{z}(r, t) \geqslant 1 \quad \text { if } t<\tilde{T} .
$$

Since $\tilde{s}(t) \rightarrow 0$ if $t \rightarrow \tilde{T}$, using (vi) of Theorem 7.1 we deduce that

$$
\tilde{z}(R, t) \geqslant 1 \quad \text { if } 0<t<\tilde{T} .
$$

Substituting this into (8.14) we obtain

$$
\tilde{T} \leqslant-\frac{\int_{\tilde{\Omega}}\left(1+\tilde{u}_{0}\right) \tilde{\phi}}{|\partial G|} .
$$

But $v \geqslant \tilde{v}$ by comparison and, consequently, $T \leqslant \tilde{T}$, so that (8.8) follows.

REMARK 8.2. If $n=1, \Omega=(0, L)$ and $u_{0}^{\prime}(x) \geqslant 0, u_{0}^{\prime \prime}(x) \geqslant 0$, then we can take $\tilde{u}_{0}=u_{0}$ and thus

$$
-\frac{1}{\left|u_{0}\right|_{\infty}} \int_{0}^{L}\left(1+u_{0}\right) \phi \leqslant T \leqslant-\int_{0}^{L}\left(1+u_{0}\right) \phi .
$$

Notice that (8.2) implies that $\left|u_{0}\right|_{\infty} \geqslant 1$. 


\section{REFERENCES}

1. L. A. Caffarelli, The regularity of free boundaries in higher dimensions, Acta Math. 139 (1977), 155-184.

2. Compactness methods in free boundary problems, Comm. Partial Differential Equations 5 (1980), 427-448.

3. _ A remark on the Hausdorff measure of a free boundary and the convergence of coincidence sets, Boll. Un. Mat. Ital. 18 (1981), 1297-1299.

4. L. A. Caffarelli and N. M. Riviere, Smoothness and analyticity of free boundaries in variational inequalities, Ann. Scuola Norm. Sup. Pisa Cl. Sci. 3 (1976), 289-310.

5. J. R. Cannon, D. B. Henry and D. B. Kotlow, Continuous differentiability of the free boundary for weak solutions of the Stefan problem, Bull. Amer. Math. Soc. 80 (1974), 45-48.

6. P. Čižek and V. Janovsky, Hele-Shaw flow model of the injection by a point source, Proc. Roy. Sci. Edinburgh Sect. A 91 (1981), 147-159.

7. A. B. Crowley, On the weak solution of moving boundary problems, J. Inst. Math. Appl. 24 (1979), 43-57.

8. C. M. Elliott, On a variational inequality formulation of an electrical machining moving boundary problem and its approximation by the finite element method, J. Inst. Math. Appl. 25 (1980), 121-131.

9. C. M. Elliott and V. Janovsky, A variational inequality approach to the Hele-Shaw flow with a moving boundary, Proc. Roy. Soc. Edinburgh Sect. A 88 (1981), 93-107.

10. C. M. Elliott and J. R. Ockendon, Weak and variational methods for moving boundary problems. Pittman, London, 1982.

11. A. Fasano and M. Primicerio, New results on some classical parabolic free boundary problems, Quart. Appl. Math. 38 (1980/81), 439-460.

12. A. Friedman, Partial differential equations of parabolic type, Prentice Hall, Englewood Cliffs, N.J., 1964.

13. Parabolic variational inequalities in unbounded domains and applications to stopping time problems, Arch. Rational Mech. Anal. 52 (1973), 134-160.

14. Analyticity of the free boundary for the Stefan problem, Arch. Rational Mech. Anal. 61 (1976), 97-125.

15. ___ Variational principles and free boundary problems, Wiley, New York, 1982.

16. A. Friedman and R. Jensen, Convexity of the free boundary in the Stefan problem and in dam problem, Arch. Rational Mech. Anal. 67 (1977), 1-24.

17. A. Friedman and D. Kinderlehrer, A one-phase Stefan problem, Indiana Univ. Math. J. 24 (1975), 1005-1035.

18. R. Jensen, The smoothness of the free boundary in the Stefan problem with supercooled water. Illinois J. Math. 22 (1978), 623-629.

19. J. A. McGeough and H. Rasmussen, On the derivation of the quasi-steady model in electrochemical machining, J. Inst. Math. Appl. 13 (1974), 13-21.

20. J. W. McLean and P. G. Saffman. The effect of surface tension on the shape of fingers in a Hele-Shaw. cell, J. Fluid Mech. 102 (1981), 455-469.

21. P. van Moerbecke, An optimal stopping problem for linear reward, Acta Math. 132 (1974), 1-41.

22. S. Richardson, Hele-Shaw flows with a free boundary produced by the injection of fluid into a narrow: channel, J. Fluid Mech. 56 (1972), 609-618.

23. Some Hele-Shaw flows with time-dependent free boundaries, J. Fluid Mech. 102 (1981), $263-278$.

24. P. G. Saffman and G. I. Taylor, The penetration of a fluid into a porous medium or Hele-Shaw cell containing a more viscous fluid, Proc. Roy. Soc. Edinburgh Sect. A 245 (1958), 312-329.

25. D. G. Schaeffer, A new proof of the infinite differentiability of the free boundary in the Stefan problem, J. Differential Equations 20 (1976), 266-269.

DEPARTMENT OF MATHEMATICS, INDIANA UNIVERSITy, BloOMINGTON, INDIANA 47401

Department of Mathematics, NorthWESTERn University, EVAnSton, Illinois 60201 